\title{
Multipath Effects on Beacon Performances
}

\author{
Ju-Lung Chu and Jean-Fu Kiang \\ Department of Electrical Engineering and \\ Graduate Institute of Communication Engineering \\ National Taiwan University \\ Taipei, Taiwan, ROC \\ jfkiang@cc.ee.ntu.edu.tw
}

\begin{abstract}
Multipath effects may create serious problems in mobile communication channels, which are caused by scattering from environments. Obvious syndromes include additional time delay, frequency shift, and fading effects. In this work, we study the multipath effects and propose a solution for alleviation.
\end{abstract}

Keywords: Multipath effects, propagation, beacon, base station.

\section{Introduction}

Intelligent transportation system(ITS) is proposed in response to the growing demands for mobility, safety, limited road capacities, and so on. There are many technologies involved in the ITS, and many applications have been proposed, such as traffic and traveler information, road guidance, parking management, and so on. Other services like electronic toll collection or access control can detour vehicles using a specific road during a specific time period. An important part of a traffic management system is the exchange of information to and from the vehicles. Communication between the infrastructure and vehicles can be carried out over roadside beacons with a short range and bidirectional data link. The range varies from several meters to several kilometers and depends on applications, vehicles and environment. In electronic toll collection application, a single beacon's coverage may be restricted to a few meters. Several systems of electronic tolling collection are mentioned in [1], [3], [4], and the ranges of those systems range from 5 to 30 meters with vehicular speed ranging from 60 to 180 kilometers per hour. In wide area network(WAN) application, the coverage area of a base station can be hundreds or thousands of meters. The optimum size of coverage area is based on both application and technology.

Performance of these applications depends on sufficiency of bandwidth to support these applications.
The data rate of a communication link is affected by many parameters such as signal modulation, encryption, interleaving, bandwidth of signals, environment of

transceivers, and so on. In this work, we will focus on the effects of environment, and analyze the difference between uplink and downlink channels. Transmission architecture will be proposed to alleviate multipath effects.

\section{Communication Environment}

The communication systems considered in this work are built for wireless, outdoor, and vehicular use. Performance of such communication links is affected by many factors, including signal modulation, encryption, interleaving, bandwidth of signals, vehicular speed, distribution of scatterers, base station distributions, geographical changes, weather, and so on. We will focus on the effects of environment. Due to wireless and outdoor environments, there are always large numbers of scattering signals. These scattering signals propagate in different paths, and results in multipath effects on the receivers. In [2], Roberson and Kaiser present a general model for path loss, carrier frequency shifts and signal time delay in a multipath environment as shown in Fig.1. They also proposed a method to reduce the power spectrum shift due to Doppler effect through carrier frequency adjustments.

In general, these three factors are analyzed and solved separately. In [5], an overfill CDM technique is proposed to improve the performance in a multipath situation with large time delays. In [6], Robertson and Kaiser analyze the perturbations caused by Doppler spreads on multi-carrier systems. However, the origin of these problems is the multipath propagation environment. Such problems might be alleviated by technical approaches. 


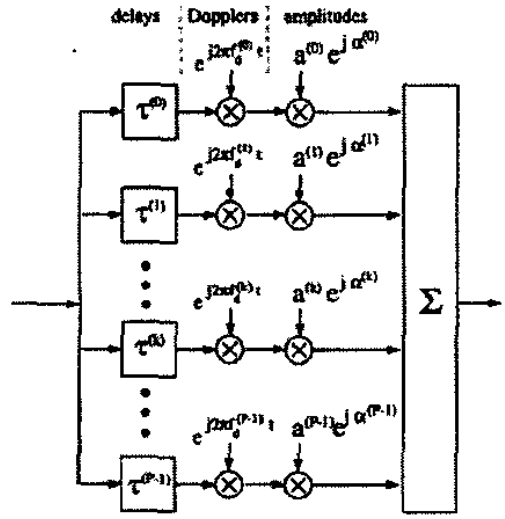

Figure 1. The channel model with $P$ paths, each with a Doppler frequency shift, attenuation and delay[2].

Relative location of scatterers to transmitter and receiver also contribute to the effects. As shown in Fig.2, the scatterers near the transmitter spread signals, and only a fraction of the spread signals can reach the receiver. Thus, it is reasonable to assume that these signal components are highly correlated.

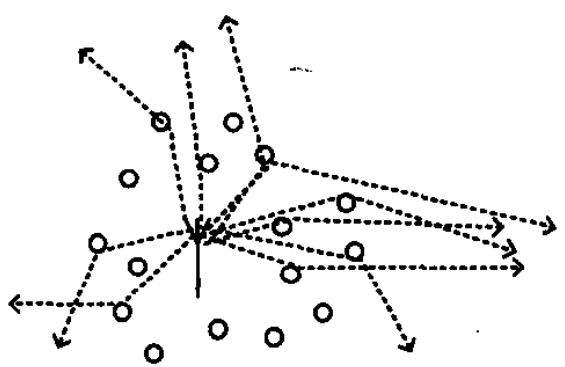

Figure 2. Scattering signals around a transmitter.

On the other hand, most scattering signals near the receiver are received, as in Fig.3. Thus, the scatterers near the receiver site are more significant in multipath effects.

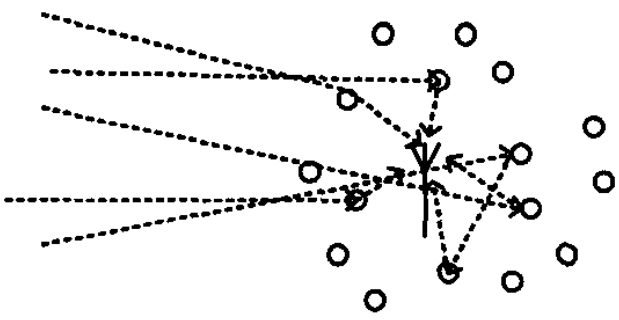

Figure 3. Scattering situation around a receiver.

Shadowing, path loss, time delays, and Doppler effects are of serious concern. These problems become less serious when the channel is time-invariant. Since a time-invariant channel can be recovered by measuring channel impulse response a priori. However, when there are relative movements between scatterers in the propagation path, things become different. Almost every parameter, like path loss, relative power, time delays Doppler frequency shift, become time variant and difficult to predict. This type of channels is called a fading channel. By the change rates of channel characteristics, fading channels can be categorized into two types: short-term and long-term fading. Short-term fading results from fast movement of transceivers. The faster the transceiver moves, the quicker the channel characteristics change. This results in serious power fluctuations at the receiver. The long-term fading mainly comes from the relative geographical change, and it changes slowly with time. The geographical changes due to the buildings and streets in the city differ only in their scales. For a low-speed vehicle moving in a city, the fading is viewed as a long-term fading, since the communication channel changes slowly over time due to small changes in its relation locations. For a high-speed vehicle, such geographical change may cause noticeable effects in performance. Fading problems and those discussed in the previous paragraphs are mainly caused by the scatterings during signal propagation. If one can construct a multipath-free zone, the speed limit of communication link and its performance can be increased at the same time.

\section{Virtual Multipath-Free Zone}

The concept of virtual multipath-free zone is presented to improve the performance of system and increase the speed limit that the system can support. Directional antennas are used in the roadside base station to restrict the signal coverage in both uplink and downlink. Fig. 4 shows the concept of directional antenna applied at overhang and roadside, respectively. Notice that the directional antennas divide multipath-free zones.

The directional antenna restricts its coverage area to the line-of-sight(LOS) path to the receiver. In the configuration shown in Fig.4, the LOS path always exists in space above vehicles. In the situations exemplified in Fig.5, the LOS path may be blocked. However, antenna diversity technique can be applied to solve such problems. The efficiency of antenna and frequency diversity techniques used in $5.8 \mathrm{GHz}$ microwave link are studied and simulated in [7]. The system can be established laneby-lane or block-by-block. Since the coverage area is restricted, the base station will not receive signals scattered from out of the coverage area. Therefore, in addition to the signal propagates through LOS path, signals scattered from other vehicles in the same area are also expected. It is reasonable to say that the smaller the 
coverage of each transceiver is, the less multipath effects will be incurred. However, other issues like high handoff rates, more base stations, frequency allocations, and so on, may be raised due to the short communication link time. Figs. 6 and 7 show some plausible antenna patterns. There are other parameters like suitable size of communication zone, transmitting power, and space diversity that need to be determined to improve the system performances.

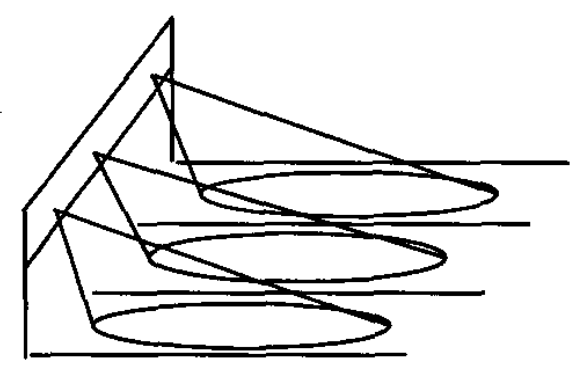

(a)

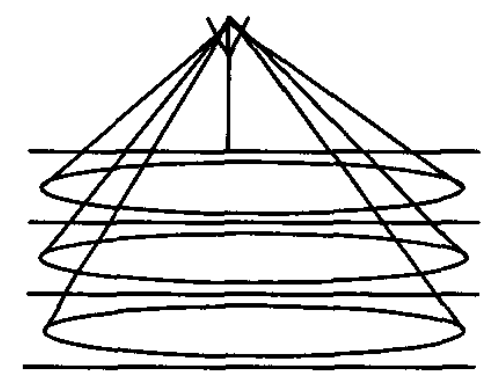

(b)

Figure 4. Beacons and communication zones for (a) overhang and (b) roadside systems.

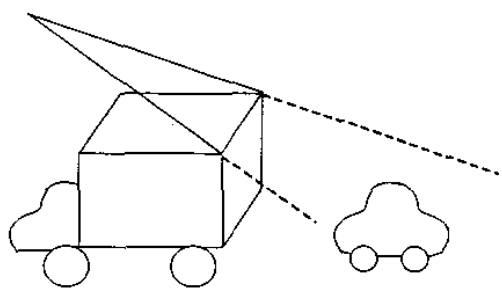

(a)

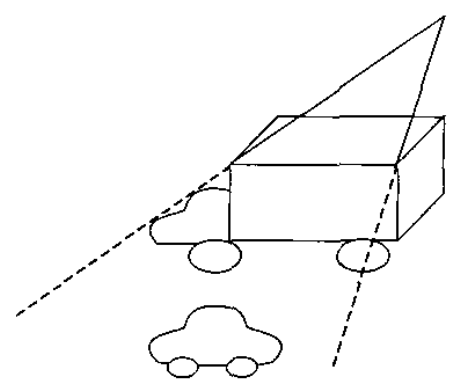

(b)

Figure 5. Possible situations of LOS blocking.

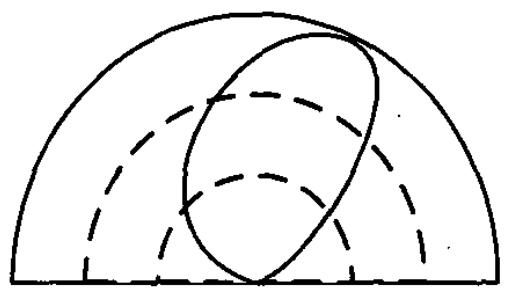

(a)

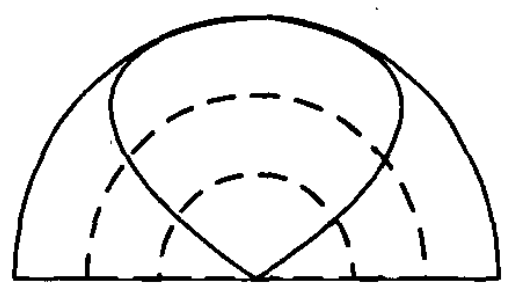

(b)

Figure 6. Side view of plausible antenna patterns.

\section{Uplink and Downlink Channels}

Information exchange systems between beacons and vehicles are the basis of most ITS applications. The communication link of an information exchange system is preferred to be reciprocal. The signal transmission from vehicles to base stations is called uplink, and that from base station to vehicle is called downlink. As mentioned before, the scatterers around the receiver site will contribute more significantly to the multipath effects. Since the base station antenna is usually stationary and mounted at higher altitude than that of vehicles, multipath signals can almost be eliminated by proper design of the receiving antenna pattern and use of space diversity 
techniques. Therefore, it is possible to construct a virtual multipath-free zone in the uplink situation. In the downlink channel, there will be some scatterers around the receiver, for example, the surrounding vehicles. In order to lower the cost of the vehicle units, simple dipole or patch antennas are proposed as the vehicle antenna. Therefore, we will focus on the base station antenna systems which are allowed to be more sophisticated.

Since the uplink of this system is expected to perform well, uplink-only or uplink-preferred services such as ETC, car flow sensing and identification can be supported. Applications such as traffic information, Internet access, and multimedia download are asymmetric in uplink and downlink data rates; their performance will also be evaluated in this work.

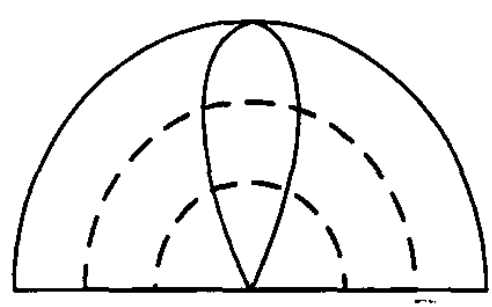

(a)

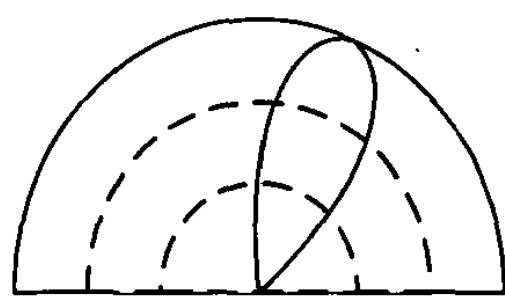

(b)

Figure 7. Top view of plausible antenna patterns.

\section{References}

[1] P. Blythe, "RFID for road tolling, road-use pricing and vehicle access control," IEE Colloq. RFID Technol., Oct. 1999.

[2] P. Robertson and S. Kaiser, "The effects of Doppler spreads in OFDM(A) mobile radio systems," Proc. IEEE Conf. Veh. Technol., pp.329-333, 1999.
[3] P. T. Blythe and P. J. Hills, "Road pricing in 5 cities: the ADEPT project," IEEE/IEE Conf . Veh. Navig. Info. Sys., pp.637-642, Oct. 1993.

[4] A. F. Dadds, "Microwave link for . data communication between a moving vehicle and a roadside beacon."

[5] K. Shimezawa, H. Harada, H. Shirai, and M. Fujise, "A high-speed DSRC system assisted by an overfill CDM for future seamless road-vehicle communication systems," Proc. IEEE Conf. Veh. Technol., vol. 3, pp.24-28, Sept. 2002.

[6] P. Robertson and S. Kaiser, "Analysis of the loss of orthogonality through Doppler spread in OFDM systems," Proc.GLOBECOM, Vol.1b, pp.701-706, 1999.

[7] W. Grabow and A. Schrei, "A study on diversity techniques in a $5.8 \mathrm{GHz}$ microwave link for traffic management systems," Proc. IEEE Intl. Symp. Pers., Indoor, Mobile Radio Commun., pp.556-560, Oct. 1992. 